\title{
Metallicity dependence of Type Ib/c and IIb supernova progenitors in binary systems
}

\author{
Sung-Chul Yoon
}

\author{
Department of Physics and Astronomy, Seoul National University, Gwanak-ro 1, Gwanak-gu, \\ Seoul, 151-742, Republic of Korea \\ email: yoon@astro.snu.ac.kr
}

\begin{abstract}
Type Ib/c supernovae ( $\mathrm{SNe} \mathrm{Ib} / \mathrm{c}$ ) are characterized by the lack of prominent hydrogen lines in thespectra, implying that their progenitors have lost most of their hydrogen envelopes by the time of the iron corecollapse. Binary interactions provide an important evolutionary chanel for $\mathrm{SNe} \mathrm{Ib} / \mathrm{c}$, and recent observations indicatethat the inferred ejecta masses of $\mathrm{SNe} \mathrm{Ibc}$ are more consistent with the prediction of the binary scenario than that ofthe single star scenario that invokes mass loss as the key evolutionary factor for $\mathrm{SNe} \mathrm{Ib} / \mathrm{c}$ progenitors. So far,theoretical predictions on the detailed properties of $\mathrm{SNe} \mathrm{Ib} / \mathrm{c}$ progenitors in binary systems have been made mostlywith models using solar metallicity. However, unlike the single star scenario, where $\mathrm{SNe} \mathrm{Ib} / \mathrm{c}$ are expected only forsufficiently high metallicity, hydrogen-deficent SN progenitors can be produced via binary interactions at anymetallicity. In this talk, I will discuss theoretical predictions on the metallicity dependence of the $\mathrm{SNe} \mathrm{Ib} / \mathrm{c}$ progenitorstructure, based on evolutionary models of massive binary stars. Sepefically, I will address how the ejecta masses ofSNe Ib and Ic and the ratio of SN Ib/c to SN IIb as well as SN Ib to SN Ic would systematically change as a function ofmetallicity, and which new types of SNe are expected in binary systems at low metallicity.
\end{abstract}

